\title{
Satisfacción del usuario externo de neurocirugía del Instituto Nacional de Ciencias Neurológicas, Lima - Perú, 2016
}

Isabel Robles Hurtado* 1,a; Maritza Placencia Medina ${ }^{2, b}$; Ricardo Carreño Escobedo ${ }^{3, c}$

RESUMEN

Objetivo: Determinar el nivel de satisfacción del usuario externo de neurocirugía del Instituto Nacional de Ciencias Neurológicas (INCN), Lima, octubre-noviembre, 2016.

Materiales y métodos: Estudio cuantitativo, descriptivo, prospectivo y transversal, en 314 usuarios. Se utilizó la encuesta SERVQUAL y se analizó la puntuación media diferencial, análisis ANCOVA y correlación de Spearman.

Resultados: La satisfacción global fue 19,4\% (61) y la insatisfacción global, 80,6 \% (253) que incluye al insatisfecho leve y moderado. En el 55,1\% (173) de adultos maduros hubo insatisfacción global. La media de las puntuaciones diferenciales para todas las dimensiones fue 0,77; fiabilidad, 0,91; capacidad de respuesta, 0,85; seguridad, 0,44; empatía, 1,03, y aspectos tangibles 0,52. Respecto a la tangibilidad, el adulto maduro es el que presenta mayor insatisfacción con 36,6 \% (115), asimismo, para la fiabilidad, 13,3\% (42); respuesta, 46,8\% (147); seguridad, 14,9\% (47); y empatía, 55,1\% (173). Hubo correlación positiva baja, estadísticamente significativa, entre el nivel de estudio del paciente y la satisfacción con la programación de citas $(r=0,185)$, entre el nivel de estudio del paciente y el respeto a la privacidad $(\mathrm{r}=0,196)$; y entre el tipo de usuario y la atención rápida de exámenes radiológicos $(r=0,184)$. Hubo correlación negativa baja estadísticamente significativa entre el nivel de estudio y la satisfacción sobre la comprensión de la explicación del neurocirujano sobre su tratamiento $(r=-0,212)$, y los carteles o letreros son adecuados para orientar $(r=-0,233)$, equipos disponibles y materiales para atención $(r=-0175)$, y limpieza de sala de espera y comodidad $(r=-0,175)$.

Conclusiones: Se encontró insatisfacción en 4 de cada 5 atendidos. Hubo correlación directa entre el nivel de estudio y programación de citas, y respeto a la privacidad. La procedencia se asocia a comprensión de la explicación del neurocirujano sobre el tratamiento.

Palabras clave: Satisfacción del paciente; Consultorios médicos; Neurocirugía (Fuente: DeCS BIREME).

\section{Satisfaction of neurosurgery outpatients of the Instituto Nacional de Ciencias Neurológicas, Lima - Peru, 2016}

\section{ABSTRACT}

Objective: To determine the satisfaction level of neurosurgery outpatients of the Instituto Nacional de Ciencias Neurológicas (INCN), Lima, from October to November 2016.

Materials and methods: A quantitative, descriptive, prospective and cross-sectional study was conducted in 314 patients. The study used the SERVQUAL survey to calculate the mean differential score, as well as the ANCOVA analysis and the Spearman's rank correlation coefficient.

1. Instituto Nacional de Ciencias Neurológicas. Lima, Perú.

2. Universidad Nacional Mayor de San Marcos. Lima, Perú.

3. Universidad de San Martín de Porres. Lima, Perú.

a. Licenciada en Enfermería.

b. Doctora de Farmacia y Bioquímica.

c. Magister en Gobierno y Gerencia de Servicios de Salud, Médico Epidemiólogo.

*Autor corresponsal

Citar como: Robles I; Placencia M; Carreño R. Satisfacción del usuario externo de neurocirugía del Instituto Nacional de Ciencias Neurológicas, Lima - Perú, 2016. Horiz Med (Lima).2019(2). 
Results: Overall satisfaction accounted for $19.4 \%$ (61) and overall dissatisfaction, $80.6 \%$ (253), which included both mild and moderate dissatisfaction. Fifty-five point one percent $(55.1 \%)$ (173) of middle-aged adults expressed overall dissatisfaction. The mean differential score for all the dimensions reached 0.77 (reliability 0.91 , responsiveness 0.85 , safety 0.44 , empathy 1.03 and tangible aspects 0.52 ). Middle-aged adults presented dissatisfaction in all dimensions: tangibility $36.6 \%(115)$, reliability $13.3 \%(42)$, responsiveness $46.8 \%(147)$, safety $14.9 \%$ (47) and empathy $55.1 \%$ (173). A statistically significant low positive correlation was observed between patients' education level and satisfaction with appointment scheduling $(r=0.185)$, patients' education level and respect for privacy $(r=0.196)$, and type of patient and fast radiological examination service $(r=0.184)$. A statistically significant low negative correlation was observed between patients' education level and satisfaction with regard to understanding the neurosurgeon's explanations of the treatment $(r=-0.212)$, adequacy of posters and signs for orientation $(r=-0.233)$, availability of equipment and materials needed for healthcare $(r=-0.175)$, and cleanliness and comfort of the waiting room $(r=-0.175)$.

Conclusions: Four (4) out of five (5) patients expressed dissatisfaction. A direct correlation was observed between patients' education level and satisfaction with appointment scheduling and respect for privacy. Patients' origin was associated with understanding the neurosurgeon's explanations of the treatment.

Keywords: Patient satisfaction; Physicians' offices; Neurosurgery (Source: MeSH NLM). 


\section{INTRODUCCIÓN}

Desde 1994, los países de las Américas han abordado el tema de calidad en los servicios de salud como parte de los procesos, lo que refleja su relevancia tanto para el sector público como para el privado ${ }^{(1)}$.

La satisfacción del usuario externo es el grado por el cual el paciente considera que el servicio de salud ha sido ejecutado por el proveedor de salud, y lo califica como útil, efectivo o que trae beneficio a su salud. Mensurar el grado de satisfacción del usuario externo neurológico cumple una función importante en la atención médica. Se trata de un juicio sobre la calidad de la atención, que incorpora las expectativas y percepciones del usuario externo (2). La satisfacción del usuario es un indicador importante de la calidad de servicios, no obstante, resulta difícil evaluarla; los métodos y las medidas para la recolección de datos suelen influir en las respuestas del usuario. Como se mencionó anteriormente, la mayoría de los usuarios afirman estar satisfechos, independientemente de la calidad real ${ }^{(3)}$.

Conocer y elevar los niveles de satisfacción en una institución de salud es una forma de asegurar la calidad de la atención, por ello debe ser una preocupación constante en todo proveedor de salud ${ }^{(4)}$.

El Instituto Nacional de Ciencias Neurológicas (INCN) es un establecimiento especializado de IV nivel de atención en salud. La evaluación del nivel de satisfacción del usuario externo de consulta de neurocirugía constituye una vía de retroalimentación para la gestión de calidad y la calidad de la atención que se brinda. La técnica empleada para obtener esta información fue la encuesta, que usa como instrumento el cuestionario tipo SERVQUAL (5), desarrollado por Parazuraman, Berry y Zeithalm (1991) ${ }^{(6)}$ para medir satisfacción del usuario externo en empresas de servicios, que luego fue adaptada y validada por Barbakus, Maugold (1992) (7) para ser aplicada en hospitales. El mismo cuestionario fue validado también en nuestro medio por Emilio Cabello en el año 2012 en el Hospital Nacional Cayetano Heredia ${ }^{(8)}$. La satisfacción y calidad en los servicios de salud del paciente están estrechamente relacionadas ${ }^{(9)}$.

Se trata de un resultado de la atención, un aspecto del bienestar psicológico del usuario externo y también un signo de éxito para la institución. La satisfacción del usuario externo asegura que cumpla su tratamiento, y lo alienta para lograr su mejoría ${ }^{(10)}$. Una evaluación con un resultado de satisfacción baja debería contribuir a plantear mejoras en la atención, además de servir como referente de atención para otros establecimientos de salud $^{(1,11,12)}$.
El INCN es un hospital de alta complejidad y el departamento de Neurocirugía atiende a pacientes que son sometidos a una intervención quirúrgica; cuenta, a su vez, con subespecialidades como neurocirugía de epilepsia, funcional, trauma, oncológica y de columna. En el año 2015, en el Departamento de Neurocirugía atendieron 6 neurocirujanos, 1 técnica de enfermería y 1 secretaria, con un total de 10349 consultas externas ${ }^{(11)}$.

El INCN es centro de referencia nacional, donde acude una diversidad de usuarios en busca de una atención de calidad, tiene 15 subespecialidades que oferta en consulta externa. Para el año 2017, brindó 82380 atenciones. Las atenciones en Neurocirugía fueron la tercera con más demanda con 6552 (7,9\%), después de Neurodegenerativas con $7679(9,3 \%) ; y$ Neurorrehabilitación, con 7120 (8,6 \%). También atienden las subespecialidades de Epilepsia, Neurología de la conducta, Neurooftalmología, Microneurocirugía, Otoneurocirugía, Neuropatología, Neurofisiología clínica, Enfermedades neurovasculares y metabólicas, Especialidades médicas, Enfermedades transmisibles en el sistema nervioso, Neuropediatría y Psiquiatría ${ }^{(12)}$.

En el INCN han realizado encuestas de satisfacción válida para los consultorios externos del INCN, con metodología SERVQUAL estandarizado del Ministerio de Salud, donde el $65,8 \%$ de usuarios externos se encontraron insatisfechos en el año 2012 ; el 59,5 \%, en el año 2013; y el 47,8 \%, en el año 2015. Esta información se encuentra publicada en la web institucional del Instituto Nacional de Ciencias Neurológicas. Dicha metodología mide la satisfacción para todos los consultorios, pero no en un consultorio externo en específico o para una subespecialidad ${ }^{(13)}$.

La métrica de la satisfacción como resultado de la atención de servicios de salud es cada vez más necesaria; disponer de estos resultados en el ámbito de la salud permite que se puedan utilizar en la práctica clínica e investigación (14).

El objetivo de este estudio fue determinar la satisfacción del usuario externo de neurocirugía del Instituto Nacional de Ciencias Neurológicas, Lima, entre octubre y noviembre de 2016, según las dimensiones de fiabilidad, capacidad de respuesta, seguridad, empatía, y aspectos tangibles.

\section{MATERIALES Y MÉTODOS}

\section{Diseño y población de estudio}

Estudiocuantitativo, descriptivo, prospectivoy transversal, en usuarios de consulta externa de Neurocirugía del Instituto Nacional de Ciencias Neurológicas en el periodo de octubre - noviembre de 2016. La unidad de análisis fue 
el usuario externo adulto. La muestra final colectada fue 314.

\section{Variables y mediciones}

El cuestionario SERVQUAL tiene elevada confiabilidad y es válido para medir la calidad de servicios de salud (15). Está demostrado que medir la satisfacción del usuario con la calidad de los servicios médicos puede predecir la aceptación de los pacientes para seguir los tratamientos y prescripciones que se les haga (16). El cuestionario SERVQUAL mide la calidad de servicio y tiene la siguiente estructura: 1) datos generales del encuestado, 2) preguntas de las expectativas del usuario externo, sobre el servicio que esperaría por recibir, en los consultorios externos del INCN, 3) preguntas sobre la percepción de la calidad de servicio recibido, y 4) ¿cuáles son las priorizaciones de la dimensión de la calidad? Las encuestas para el servicio de Consulta Externa (17) incluyen, en su estructura, 22 preguntas de expectativas y 22 preguntas de percepciones, distribuidas en cinco criterios o dimensiones de evaluación de la calidad que son fiabilidad (preguntas 1 a 5), capacidad de respuesta (preguntas 6 a 9), seguridad(preguntas 10 a 13), empatía (preguntas 14 a 18), y aspectos tangibles (preguntas 19 a 22). Las áreas de preguntas (expectativas y percepción) están diseñadas al tipo de escala de Likert del 1 al 7; que considera 1 a la más baja y 7 , a la más alta.

El grado de satisfacción de los usuarios externos se determinará al calcular la diferencia entre las respuestas brindadas para las expectativas y las percepciones; posteriormente se interpretará según los siguientes parámetros: menor de $0=$ Muy Satisfecho, $0=$ Satisfecho, mayor que 0 y menor o igual a 2 = Insatisfacción leve, y mayor que 2 = Insatisfacción moderada. Los criterios de inclusión fueron ser usuario de consultorios externos de Neurocirugía del INCN, de ambos sexos, con edad mayor o igual a 18 años, que no tengan ninguna alteración de conciencia y sea capaz de comunicarse en español, que haya aceptado voluntariamente participar en el estudio luego de recibir la información verbal de las características y objetivos de este $y$, finalmente, los usuarios externos nuevos o continuadores en el servicio. Se excluyeron los usuarios menores de 18 años y provenientes de otras especialidades.

\section{Análisis estadístico}

Se utilizó el programa estadístico SPSS 20, el análisis descriptivo, a través de medidas de resumen como media, mediana, cálculo de frecuencias simples, absolutas y relativas y desviación estándar. Se halló la media de la puntuación diferencial SERVQUAL, se realizó análisis ANCOVA y correlación de Spearman.

El estudio fue autorizado por el Comité de Ética e Investigación del Instituto Nacional de Ciencias Neurológicas, y se utilizó el consentimiento informado en cada paciente. La recolección de datos estuvo programada para el periodo octubre - noviembre del 2016. Se usó como instrumento el cuestionario tipo SERVQUAL, desarrollado por Parazuraman, A. Berry L. y Zeithalm (1991), para medir satisfacción del usuario externo en empresas de servicios, luego adaptada y validada por Barbakus, Maugold (1992) para ser aplicada en hospitales. El mismo cuestionario fue validado también en nuestro medio en el 2012, por Emilio Cabello, en el Hospital Nacional Cayetano Heredia.

\section{RESULTADOS}

Se encuestaron 314 usuarios externos, 158 mujeres $(50,3 \%)$ y 156 varones $(49,7 \%)$. La muestra colectada tuvo una edad promedio de 47,5 $\pm 16,1$ años, con un rango de edad de 18 - 89 años. El grupo etario entre 50 a 59 años fue el más numeroso con 74 pacientes $(23,6 \%)$. Según el nivel de estudios, 167 participantes $(53,2 \%)$ tenían estudios secundarios, y 11 (4\%) fueron analfabetos. Del total de encuestados, 219 usuarios externos $(69,7 \%)$ contaban con el seguro integral de salud (SIS). Según tipo de usuario, 245 (78 \%) fueron continuadores. 182 usuarios $(58,0 \%)$ procedían de Lima, y 132 (4,0\%) de provincia (Tabla 1$)$.

Tabla 1. Datos sociodemográficos de usuarios externos de consulta de Neurocirugía. Octubre- noviembre 2016

\begin{tabular}{|lcc}
\multicolumn{3}{c}{$\begin{array}{c}\text { Características sociodemográficas de usuarios } \\
\text { de consultorio de Neurocirugía }\end{array}$} \\
\hline Sexo & $\mathbf{n}$ & $\%$ \\
\hline Femenino & 158 & 50,3 \\
\hline Masculino & 156 & 49,7 \\
\hline Total & 314 & 100 \\
Edad & $\mathbf{n}$ & $\%$ \\
\hline $18-19$ & 6 & 1,9 \\
\hline $20-29$ & 52 & 16,6 \\
\hline
\end{tabular}

\begin{tabular}{|lcc|}
\hline \multicolumn{3}{|c}{ Características sociodemográficas de usuarios } \\
de consultorio de Neurocirugía \\
\hline $30-39$ & 41 & 13,1 \\
$40-49$ & 61 & 19,4 \\
\hline $50-59$ & 74 & 23,6 \\
$60-69$ & 57 & 18,2 \\
\hline $70-79$ & 17 & 5,4 \\
$80-$ más & 6 & 1,9 \\
\hline Total & 314 & 100 \\
\hline
\end{tabular}


Satisfacción del usuario externo de neurocirugía del Instituto Nacional de Ciencias Neurológicas, Lima - Perú, 2016

\begin{tabular}{|c|c|c|}
\hline \multicolumn{3}{|c|}{$\begin{array}{c}\text { Características sociodemográficas de usuarios } \\
\text { de consultorio de Neurocirugía }\end{array}$} \\
\hline Nivel de estudios & $\mathbf{n}$ & $\%$ \\
\hline Analfabeto & 11 & 3,5 \\
\hline Primaria & 57 & 18,2 \\
\hline Secundaria & 167 & 53,2 \\
\hline Superior & 79 & 25,2 \\
\hline Total & 314 & 100 \\
\hline Tipo de seguro & $\mathbf{n}$ & $\%$ \\
\hline SIS & 219 & 69,7 \\
\hline SOAT & 5 & 1,6 \\
\hline Ninguno & 0 & 0,0 \\
\hline Otro & 90 & 28,7 \\
\hline Total & 314 & 100 \\
\hline
\end{tabular}

La satisfacción global fue 19,4\% (61), esto involucra a los niveles muy satisfecho y satisfecho. La insatisfacción global fue $80,6 \%$ (253), que incluye a insatisfecho leve y moderado. El 55,1 \% (173) de adultos maduros reportó insatisfacción

\begin{tabular}{|lcc|}
\multicolumn{3}{|c}{$\begin{array}{c}\text { Características sociodemográficas de usuarios } \\
\text { de consultorio de Neurocirugía }\end{array}$} \\
\hline Tipo de usuario & $\mathbf{n}$ & $\%$ \\
\hline Nuevo & 69 & 22,0 \\
Continuador & 245 & 78,0 \\
\hline Total & $\mathbf{3 1 4}$ & $\mathbf{1 0 0}$ \\
\hline Procedencia & $\mathbf{n}$ & $\%$ \\
\hline Lima & 182 & 58,0 \\
\hline Provincia & 132 & 42,0 \\
\hline Total & 314 & 100 \\
\hline Edad en años (rango) & $\mathbf{4 7 , 5 \pm 1 6 , 1}$ & $\mathbf{( 1 8 - 8 9 )}$ \\
\hline
\end{tabular}

global. Respecto a la tangibilidad el adulto maduro es el que presenta mayor insatisfacción con 36,6 \% (115), del mismo modo para la fiabilidad con $13,3 \%$ (42), respuesta $46,8 \%$ (147), seguridad 14,9\% (47) y empatía 55,1 \% (173) (Tabla 2).

Tabla 2. Niveles de satisfacción global, según dimensiones y grupo etario

\begin{tabular}{|c|c|c|c|c|c|c|c|c|c|c|c|}
\hline \multirow[t]{2}{*}{ Dimensión } & \multirow[t]{2}{*}{$\begin{array}{l}\text { Grupo } \\
\text { etario }\end{array}$} & \multicolumn{2}{|c|}{$\begin{array}{c}\text { Muy } \\
\text { satisfecho }\end{array}$} & \multicolumn{2}{|c|}{ Satisfecho } & \multicolumn{2}{|c|}{$\begin{array}{c}\text { Insatisfecho } \\
\text { leve }\end{array}$} & \multicolumn{2}{|c|}{$\begin{array}{l}\text { Insatisfecho } \\
\text { moderado }\end{array}$} & \multicolumn{2}{|c|}{ Total } \\
\hline & & $\mathbf{N}$ & $\%$ & $\mathbf{N}$ & $\%$ & $\mathbf{N}$ & $\%$ & $\mathbf{N}$ & $\%$ & $\mathbf{N}$ & $\%$ \\
\hline \multirow[t]{4}{*}{ Global } & Adulto joven & 8 & 2,5 & 8 & 2,5 & 34 & 10,8 & 11 & 3,5 & 61 & 19,4 \\
\hline & Adulto maduro & 27 & 8,6 & 13 & 4,1 & 139 & 44,3 & 34 & 10,8 & 213 & 67,8 \\
\hline & Adulto mayor & 4 & 1,3 & 1 & 0,3 & 27 & 8,6 & 8 & 2,5 & 40 & 12,7 \\
\hline & Total & 39 & 12,4 & 22 & 7,0 & 200 & 63,7 & 53 & 16,9 & 314 & 100,0 \\
\hline \multirow[t]{4}{*}{ Tangibilidad } & Adulto joven & 22 & 7,0 & 14 & 4,5 & 20 & 6,4 & 5 & 1,6 & 61 & 19,4 \\
\hline & Adulto maduro & 52 & 16,6 & 46 & 14,6 & 85 & 27,1 & 30 & 9,6 & 213 & 67,8 \\
\hline & Adulto mayor & 8 & 2,5 & 6 & 1,9 & 20 & 6,4 & 6 & 1,9 & 40 & 12,7 \\
\hline & Total & 82 & 26,1 & 66 & 21,0 & 125 & 39,8 & 41 & 13,1 & 314 & 100,0 \\
\hline \multirow[t]{4}{*}{ Fiabilidad } & Adulto joven & 17 & 5,4 & 33 & 10,5 & 9 & 2,9 & 2 & 0,6 & 61 & 19,4 \\
\hline & Adulto maduro & 69 & 22,0 & 102 & 32.5 & 39 & 12,4 & 3 & 1,0 & 213 & 67,8 \\
\hline & Adulto mayor & 16 & 5,1 & 17 & 5,4 & 7 & 2,2 & 0 & 0,0 & 40 & 12,7 \\
\hline & Total & 102 & 32,5 & 152 & 48,4 & 55 & 17,5 & 5 & 1,6 & 314 & 100,0 \\
\hline \multirow[t]{4}{*}{ Respuesta } & Adulto joven & 7 & 2,2 & 6 & 1,9 & 34 & 10,8 & 14 & 4,5 & 61 & 19,4 \\
\hline & Adulto maduro & 34 & 10,8 & 32 & 10,2 & 105 & 33,4 & 42 & 13,4 & 213 & 67,8 \\
\hline & Adulto mayor & 7 & 2,2 & 3 & 1,0 & 22 & 7,0 & 8 & 2,5 & 40 & 12,7 \\
\hline & Total & 48 & 15,3 & 41 & 13,1 & 161 & 51,3 & 64 & 20,4 & 314 & 100,0 \\
\hline \multirow[t]{2}{*}{ Seguridad } & Adulto joven & 14 & 4,5 & 17 & 5,4 & 23 & 7,3 & 7 & 2,2 & 61 & 19,4 \\
\hline & Adulto maduro & 77 & 24,5 & 89 & 28,3 & 35 & 11,1 & 12 & 3,8 & 213 & 67,8 \\
\hline
\end{tabular}




\begin{tabular}{|c|c|c|c|c|c|c|c|c|c|c|c|}
\hline \multirow[t]{2}{*}{ Dimensión } & \multirow[t]{2}{*}{$\begin{array}{l}\text { Grupo } \\
\text { etario }\end{array}$} & \multicolumn{2}{|c|}{$\begin{array}{c}\text { Muy } \\
\text { satisfecho }\end{array}$} & \multicolumn{2}{|c|}{ Satisfecho } & \multicolumn{2}{|c|}{$\begin{array}{c}\text { Insatisfecho } \\
\text { leve }\end{array}$} & \multicolumn{2}{|c|}{$\begin{array}{l}\text { Insatisfecho } \\
\text { moderado }\end{array}$} & \multicolumn{2}{|c|}{ Total } \\
\hline & & $\mathbf{N}$ & $\%$ & $\mathbf{N}$ & $\%$ & $\mathbf{N}$ & $\%$ & $\mathbf{N}$ & $\%$ & $\mathbf{N}$ & $\%$ \\
\hline & Adulto mayor & 12 & 23,8 & 27 & 8,6 & 1 & 0,3 & 0 & 0,0 & 40 & 12,7 \\
\hline & Total & 103 & 32,8 & 133 & 42,4 & 59 & 18,8 & 19 & 6,1 & 314 & 100,0 \\
\hline \multirow[t]{4}{*}{ Empatía } & Adulto joven & 8 & 2,5 & 8 & 2,5 & 34 & 10,8 & 11 & 3,5 & 61 & 19,4 \\
\hline & Adulto maduro & 27 & 8,6 & 13 & 4,1 & 139 & 44,3 & 34 & 10,8 & 213 & 67,8 \\
\hline & Adulto mayor & 4 & 1,3 & 1 & 0,3 & 27 & 8,6 & 8 & 2,5 & 40 & 12,7 \\
\hline & Total & 39 & 12,4 & 22 & 7,0 & 200 & 63,7 & 53 & 16,9 & 314 & 100,0 \\
\hline
\end{tabular}

La media de las puntuaciones diferenciales entre expectativas y percepciones para todas las dimensiones fue 0,77 . En la fiabilidad fue 0,91; capacidad de respuesta,
0,85; seguridad, 0,44; empatía, 1,03; y aspectos tangibles, 0,52 (Figura 1).

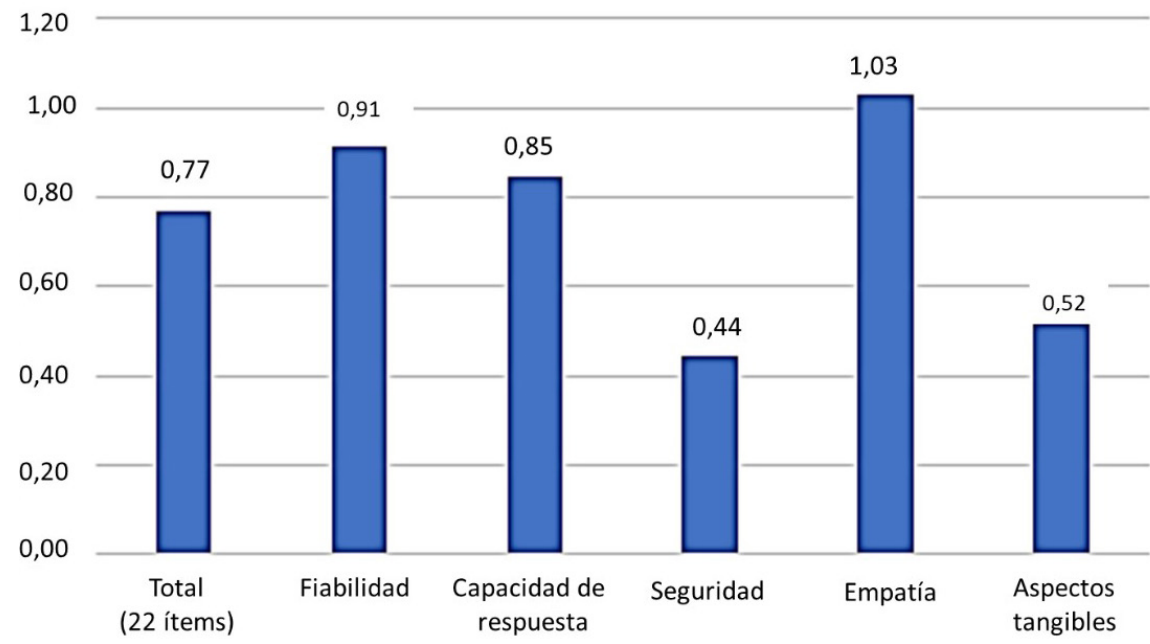

Figura 1. Media de las puntuaciones diferenciales total y según dimensiones

Las puntuaciones diferenciales obtenidas por los pacientes en los items, según dimensiones y la escala SERVQUAL global

Las medias de las puntuaciones diferenciales obtenidas en los 22 ítems de la escala SERVQUAL se muestran en la tabla 3. La puntuación más baja $(-0,06)$ corresponde al ítem número 1 , relativo a la valoración de la orientación recibida por el personal de informes, es el único ítem con valoración negativa, y nos indica un nivel "muy satisfecho". Los demás ítems tienen valoración positiva, lo que nos señala que la satisfacción es "insatisfecho leve", ya que no excede el valor de 2 . También se muestran los estadísticos descriptivos relativos a las puntuaciones diferenciales SERVQUAL obtenidas en las 5 dimensiones, la puntuación global y la puntuación de los 22 ítems y la desviación estándar. La puntuación de las 5 dimensiones fue positiva, lo que indica que, en cada dimensión, el nivel de satisfacción fue de "insatisfecho leve", es decir, la perspectiva es menor a la expectativa.

La mayor puntuación de la media diferencial según dimensiones corresponde a la dimensión empatía $(1,03)$, y la más baja, a la dimensión tangibilidad $(0,52)$. Todas indican insatisfacción leve. La puntuación diferencial global media de la escala SERVQUAL o satisfacción global fue 0,77 (Tabla 3 ). 
Tabla 3. Puntuación media diferencial SERVQUAL

\begin{tabular}{|c|c|c|c|}
\hline & Media & Desv. típ. & Satisfacción \\
\hline D.1 Personal de informes orientó & $-0,06$ & 0,97 & Muy satisfecho \\
\hline D.2 Consulta de neurocirujano en horario programado & 0,47 & 1,39 & Insatisfecho leve \\
\hline D.3 Se respetó la programación & 1,19 & 1,32 & Insatisfecho leve \\
\hline D.4 Disponibilidad de historia clínica & 1,36 & 1,37 & Insatisfecho leve \\
\hline D.5 Citas disponibles y obtenidas con facilidad & 1,61 & 1,23 & Insatisfecho leve \\
\hline D.6 Atención módulo SIS rápida & 1,25 & 1,41 & Insatisfecho leve \\
\hline D.7 Atención análisis de laboratorio rápida & 0,54 & 1,43 & Insatisfecho leve \\
\hline D.8 Atención exámenes radiológicos rápida & 0,35 & 1,38 & Insatisfecho leve \\
\hline D.9 Atención farmacia rápida & 1,25 & 1,47 & Insatisfecho leve \\
\hline D.10 Respeto a privacidad & 0,10 & 1,31 & Insatisfecho leve \\
\hline D.11 Examen físico completo y minucioso & 0,40 & 1,53 & Insatisfecho leve \\
\hline D.12 Médico brindó tiempo necesario para sus dudas & 1,07 & 1,44 & Insatisfecho leve \\
\hline D.13 Médico inspira confianza & 0,20 & 1,31 & Insatisfecho leve \\
\hline D.14 Personal neurocirugía con trato amable & 1,35 & 1,29 & Insatisfecho leve \\
\hline D.15 Médico se interesó en solucionar su problema de salud & 0,57 & 1,55 & Insatisfecho leve \\
\hline D.16 Comprendió la explicación del neurocirujano sobre su salud & 0,98 & 1,22 & Insatisfecho leve \\
\hline D.17 Comprendió la explicación del neurocirujano sobre su tratamiento & 1,22 & 1,31 & Insatisfecho leve \\
\hline D.18 Comprendió la explicación del neurocirujano sobre procedimientos 0 análisis & 1,03 & 1,45 & Insatisfecho leve \\
\hline D.19 Los carteles o letreros son adecuados para orientar & 0,70 & 1,51 & Insatisfecho leve \\
\hline D.20 Personal para informar al paciente y acompañantes & 0,08 & 1,46 & Insatisfecho leve \\
\hline D.21 Equipos disponibles y materiales para atención & 0,25 & 1,42 & Insatisfecho leve \\
\hline D.22 Limpieza de sala de espera y comodidad & 1,04 & 1,35 & Insatisfecho leve \\
\hline Puntuación diferencial de tangibilidad & 0,52 & 0,25 & Insatisfecho leve \\
\hline Puntuación diferencial de fiabilidad & 0,91 & 0,20 & Insatisfecho leve \\
\hline Puntación diferencial de respuesta & 0,85 & 0,06 & Insatisfecho leve \\
\hline Puntuación diferencial de seguridad & 0,44 & 0,28 & Insatisfecho leve \\
\hline Puntuación diferencial de empatía & 1,03 & 0,31 & Insatisfecho leve \\
\hline Puntuación diferencial con 22 ítems & 0,77 & 0,07 & Insatisfecho leve \\
\hline
\end{tabular}

\section{Análisis de correlación}

Al analizar la correlación de la satisfacción con la atención en consulta externa por neurología se encontró los siguientes hallazgos estadísticamente significativos:

Edad del paciente: Hubo una correlación inversa entre la edad del paciente y que el médico inspire confianza $(-0,149)$, una correlación directa entre la edad del paciente y personal neurocirugía con trato amable $(0,126)$ y una correlación directa entre la edad del paciente y si comprendió la explicación del neurocirujano sobre su tratamiento $(0,125)$.
Sexo del paciente: Encontramos una correlación positiva entre el sexo masculino con la limpieza de sala de espera y comodidad.

Nivel de estudio: Aparece una correlación positiva entre el nivel de estudio del paciente y la satisfacción con la programación de citas $(0,185)$, entre el nivel de estudio y el respeto a la privacidad $(0,196)$. Se halló correlación negativa entre el nivel de estudio y si comprendió la explicación del neurocirujano sobre su tratamiento $(-0,212)$. En relación a si los carteles o letreros son adecuados para orientar $(-0,233)$, equipos disponibles $y$ materiales para atención $(-0,175)$, y limpieza de sala de 
espera y comodidad $(-0,175)$.

Tipo de usuario: Hubo correlación negativa entre tipo de usuario y satisfacción con el personal que brindó informes u orientó $(-0,112)$, los equipos disponibles y materiales para atención $(-0,111)$, y la limpieza de sala de espera y comodidad $(-0,136)$. Encontramos una correlación positiva respecto a la consulta del neurocirujano en horario programado $(0,123)$, a la atención módulo SIS rápida $(0,139)$, la atención exámenes radiológicos rápida $(0,184)$, atención farmacia rápida $(0,153)$, el respeto a privacidad $(0,138)$, y el examen físico completo y minucioso $(0,112)$ (Tabla 4).

Tabla 4. Correlación de Spearman sobre la satisfacción con la atención en consulta externa por neurología

\begin{tabular}{|c|c|c|c|c|c|}
\hline & Edad & Sexo & $\begin{array}{l}\text { Nivel de } \\
\text { estudio }\end{array}$ & Seguro & $\begin{array}{l}\text { Tipo de } \\
\text { usuario }\end{array}$ \\
\hline Satisfacción con el personal que brindó informes u orientó & -.020 & -.078 & -.091 & -.078 &,$- 112^{*}$ \\
\hline Satisfacción consulta de neurocirujano en horario programado & -.034 & -.007 & .104 & .022 & $123^{*}$ \\
\hline Satisfacción con la programación & -.021 & -.044 &, $185^{\star *}$ & .008 & .091 \\
\hline Satisfacción con la disponibilidad de historia clínica & -.042 & .053 & -.074 & -.086 & .045 \\
\hline Satisfacción con las citas disponibles y obtenidas con facilidad & -.090 & -.013 & .030 & -.030 & .045 \\
\hline Atención módulo SIS rápida & -.071 & .067 & .080 & -.006 & $139 *$ \\
\hline Atención análisis de laboratorio rápida & -.070 & -.043 & .040 & -.005 & -.011 \\
\hline Atención exámenes radiológicos rápida & -.067 & -.044 &, $195^{\star *}$ & .060 & $184^{\star *}$ \\
\hline Atención farmacia rápida & -.001 & -.045 & -.001 & -.023 & $153^{\star *}$ \\
\hline Respeto a privacidad & -.088 & -.012 & $196^{* *}$ & .043 &, $138^{*}$ \\
\hline Examen físico completo y minucioso & -.065 & -.022 & .089 & -.077 &, $112^{*}$ \\
\hline Médico brindó tiempo necesario para sus dudas & -.005 & .035 & -.015 & .079 & -.029 \\
\hline Médico inspira confianza &,$- 149^{\star *}$ & -.061 & .018 & -.100 & -.047 \\
\hline Personal neurocirugía con trato amable &, $126^{*}$ & -.040 & -.097 & .086 & -.006 \\
\hline Médico se interesó en solucionar su problema de salud & .059 & -.037 & .003 & .038 & -.001 \\
\hline Comprendió la explicación del neurocirujano sobre su salud & .042 & -.053 & -.018 & .024 & -.027 \\
\hline Comprendió la explicación del neurocirujano sobre su tratamiento & $125^{*}$ & .093 &,$- 212^{\star *}$ & -.049 & -.031 \\
\hline Comprendió la explicación del neurocirujano sobre procedimientos o análisis & -.022 & -.021 & .073 & -.064 & .103 \\
\hline Los carteles o letreros son adecuados para orientar & .092 & .053 &,$- 233^{\star *}$ & -.002 & .021 \\
\hline Personal para informar al paciente y acompañantes & -.014 & -.057 & .095 & -.011 & .019 \\
\hline Equipos disponibles y materiales para atención & ,111* & .034 &,$- 175^{\star *}$ & .062 &,$- 111^{*}$ \\
\hline Limpieza de sala de espera y comodidad & .102 &, $114^{*}$ &,$- 175^{\star *}$ & .060 &,$- 136^{*}$ \\
\hline
\end{tabular}

** La correlación Spearman es significativa al nivel 0,01 (bilateral).

* La correlación Spearman es significativa al nivel 0,05 (bilateral).

Se encontró correlación positiva baja estadísticamente significativa para la satisfacción en la atención en consulta externa por neurología para las siguientes variables: entre la edad del paciente y el personal neurocirugía con trato amable $(0,126)$, entre la edad del paciente y si comprendió la explicación del neurocirujano sobre su tratamiento $(0,125)$, entre el sexo masculino con la limpieza de sala de espera y comodidad $(0,114)$, y entre el nivel de estudio del paciente y la satisfacción con la programación de citas $(0,185)$, así como con el respeto a la privacidad $(0,196)$; entre el tipo de usuario y la satisfacción en consulta de neurocirujano en horario programado $(0,123)$, así como en la atención módulo SIS rápida $(0,139)$, la atención exámenes radiológicos rápida $(0,184)$, atención farmacia rápida $(0,153)$, respeto a privacidad $(0,138)$, y examen físico completo y minucioso $(0,112)$.

Los hallazgos con una correlación negativa baja estadísticamente significativa entre la satisfacción en la atención en consulta externa por neurología que se encontraron fueron entre la edad del paciente y que 
el médico inspire confianza $(-0,149)$; entre el nivel de estudio y si comprendió la explicación del neurocirujano sobre su tratamiento $(-0,212)$, y los carteles o letreros son adecuados para orientar $(-0,233)$; los equipos disponibles y materiales para atención $(-0,175)$, y la limpieza de sala de espera y comodidad $(-0,175)$. En tipo de usuario y satisfacción con el personal que brindó informes u orientó $(-0,112)$ con los equipos disponibles y materiales para atención $(-0,111)$, y con la limpieza de sala de espera y comodidad $(-0,136)$.

En el análisis ANCOVA, las variables predictivas relacionadas con la puntuación diferencial SERVQUAL son el 17,1 \% de la variación del respeto a la privacidad se explica por la procedencia. La media de la diferencia de satisfacción es mayor en los de provincia. La procedencia se asocia en $15,7 \%$ para la diferencia entre percepciones y expectativas sobre el respeto a la privacidad, la procedencia se asocia en $11,1 \%$ para la diferencia entre percepciones y expectativas para la comprensión de la explicación del neurocirujano sobre su tratamiento. El $12,4 \%$ de la variación del respeto a la satisfacción por la comprensión de la explicación del neurocirujano sobre su tratamiento se explica por la procedencia, eliminando la influencia de la edad. La media de la diferencia de satisfacción de la comprensión de la explicación del neurocirujano sobre su tratamiento es mayor en Lima comparado con provincia $1,5$ [IC $95 \%, 1,414-1,774]$.

Análisis ANCOVA de los puntajes diferenciales de satisfacción - Variables predictivas relacionadas con la puntuación diferencial SERVQUAL (Tabla 4)

La diferencial de satisfacción en la comprensión de la explicación del neurocirujano sobre su tratamiento como variable dependiente, como independiente el sexo, y edad como covariable. La edad está asociada a la diferencial de satisfacción como comprensión de la explicación del neurocirujano sobre su tratamiento ( $p$ valor 0,05 ) pero, una vez descontado este hecho, el sexo sí revela un efecto estadísticamente significativo; hay diferencia entre hombres y mujeres una vez descontada la edad. En relación a la comprensión de la explicación del neurocirujano sobre su tratamiento, podemos plantear lo siguiente, el 1,3\% de la varianza está relacionada al sexo del usuario; la varianza total del mismo ítem es baja $(2,7 \%)$ dado que hemos colocado como covariable la edad vinculada a la comprensión de la explicación del neurocirujano. La media de la comprensión de la explicación del neurocirujano sobre su tratamiento es más alta en los varones $(1,3)$ [IC $95 \%, 1,167-1,576]$, que en las mujeres $(1,0)$ [IC $95 \%$, $0,873-1,279]$. Estas medias ya están corregidas, se han descontado las diferencias en la edad en ambos grupos. Por tanto, hay diferencias estadísticas significativas y se obtienen mayores indicadores de comprensión de la explicación del neurocirujano sobre su tratamiento (medias) para los varones una vez que se ha descontado la diferencia en la edad en función del sexo.

La diferencial de satisfacción en la limpieza de sala de espera y comodidad como variable dependiente, como independiente el sexo y edad como covariable. La edad está asociada a la diferencial de satisfacción como limpieza de sala de espera y comodidad ( $p$ valor 0,029 ), pero, una vez descontado este efecto, el sexo sí revela un efecto estadísticamente significativo ( $p$ valor 0,032 ), hay diferencia entre hombres y mujeres una vez que se ha descontado la edad. El $1,5 \%$ de la varianza de la limpieza de sala de espera y comodidad está relacionado al sexo del participante. La varianza total de limpieza de sala de espera y comodidad es baja $(2,0 \%)$, porque hemos colocado como covariable la edad vinculada a la limpieza de sala de espera y comodidad. La media de la diferencial entre percepción y expectativa para la limpieza de sala de espera y comodidad es más alta en los varones $(1,2)$ [IC $95 \%, 0,994-1,418]$, que en las mujeres $(0,8)$ [IC $95 \%$, $0,668-1,089]$. Estas medias ya están corregidas (se han descontado las diferencias en la edad en ambos grupos). Por tanto, hay diferencias estadísticas significativas y se obtienen mayores indicadores de limpieza de sala de espera y comodidad (medias) para los varones, una vez que se ha descontado la diferencia en la edad en función del sexo.

El nivel de estudio explica el 5,3\% de las varianzas de las diferenciales de las puntuaciones entre percepciones y expectativas, cuando se respeta la programación de atenciones, con una media mayor en los que tienen estudios superiores 1,6 [IC 95\%, 1,380 - 1,980]. Igualmente, explica el 3,2 \% de las diferenciales de puntuaciones entre percepción y expectativa, para la variable dependiente atención de exámenes radiológicos rápido. La media más alta es de nivel superior con 0,7 pero no significativa.

La diferencia entre las percepciones y expectativas en relación a la privacidad, se explica por el nivel de estudio en $4,1 \%$. El $5 \%$ de la variación del respeto a la privacidad se debe al nivel de estudio. La media del nivel estudio superior es mayor que los otros niveles con ,556 [IC $95 \%$, $0,259-0,853]$. El nivel de estudio se asocia en 3,8 \% para la diferencia entre percepciones y expectativas sobre la comprensión de la explicación del neurocirujano sobre su tratamiento. El 4,7 \% de la variación del respeto a la privacidad se debe al nivel de estudio.

El 1,5\% de la varianza de la diferencia entre percepción y expectativas respecto a si los carteles o letreros son adecuados para orientar está asociado a la edad. La varianza total de la satisfacción con este ítem es baja $(4,8 \%)$, dado que hemos colocado como covariable la edad 
vinculada a los carteles o letreros adecuados para orientar. La media de la diferencial entre percepción y expectativa para los carteles o letreros adecuados para orientar es más alta para el nivel de estudios de primaria $(1,4)$ [IC $95 \%, 1,001$ $1,815]$, que en los otros niveles de estudio. Estas medias ya están corregidas luego de descontar las diferencias en la edad en los diferentes niveles de estudio. Entonces, hay diferencias estadísticas significativas y se obtienen mayores medias de diferencial para los carteles o letreros adecuados para orientar en el nivel de estudio primaria, y se elimina la influencia de la edad en función del nivel de estudio.

En lo que respecta a la procedencia, esta se relaciona en $15,7 \%$ a la diferencia entre percepciones y expectativas sobre el respeto a la privacidad. Asimismo, el $17,1 \%$ de la variación del respeto a la privacidad se explica por la procedencia. La media de la diferencia de satisfacción es mayor en los de provincia. Por otro lado, la procedencia se asocia en 5,4 \% para la diferencia entre percepciones y expectativas sobre el examen físico completo y minucioso, y explica el $5,4 \%$ de la variación del respeto a la satisfacción por el examen físico completo y minucioso. La media de la diferencia de satisfacción al examen físico completo y minucioso es mayor en los participantes de provincia.

Además, la procedencia, en 11,1 \% está asociada para la diferencia entre percepciones y expectativas para la comprensión de la explicación del neurocirujano sobre su tratamiento. El $12,4 \%$ de la variación del respeto a la satisfacción por la comprensión de la explicación del neurocirujano sobre su tratamiento se explica por la procedencia, y elimina la influencia de la edad. La media de la diferencia de satisfacción de la comprensión de la explicación del neurocirujano sobre su tratamiento es mayor en Lima comparado con provincia 1,5 [IC 95 \%, 1,414 - 1,774]. En relación a otros aspectos de la encuesta, la procedencia se asocia en $4,2 \%$ para la diferencia entre percepciones y expectativas cuando hay equipos disponibles y materiales para atención. El 5,2 \% de la variación del respeto a la satisfacción para con los equipos disponibles y materiales para atención se explica por la procedencia, lo que elimina la influencia de la edad. La media de la diferencia de satisfacción para con los equipos disponibles y materiales para atención es mayor en Lima comparado con provincia 0,4 [IC $95 \%, 0.291$ 0.697 . Finalmente, la procedencia se asocia en 5,9\% para la diferencia entre percepciones y expectativas cuando hay limpieza de sala de espera y comodidad. El $6,4 \%$ de la variación del respeto a la satisfacción con la limpieza de sala de espera y comodidad, se explica por la procedencia, y elimina la influencia de la edad. La media de la diferencia de satisfacción para la limpieza de sala de espera y comodidad es mayor en Lima comparado con provincia 1,3 [IC $95 \%, 1.131$ - 1.515] (Tabla 5).

Tabla 5. Variables predictivas relacionadas con la puntuación diferencial SERVQUAL

\begin{tabular}{|c|c|c|c|c|}
\hline Según & Variable predictiva & $\begin{array}{l}\text { Puntaje SERVQUAL } \\
\text { (IC del } 95 \% \text { ) }\end{array}$ & $\mathrm{Eta}^{2}$ & $\mathbf{p}$ \\
\hline \multirow[t]{6}{*}{ Sexo } & Comprendió la explicación del neurocirujano sobre su tratamiento * & & & \\
\hline & Femenino & $0,873-1,279$ & 0,03 & 0,005 \\
\hline & Masculino & $1,167-1,576$ & & \\
\hline & Limpieza de sala de espera y comodidad* & & & \\
\hline & Femenino & $0,668-1,089$ & 0,03 & 0,016 \\
\hline & Masculino & $0,994-1,418$ & & \\
\hline Grado de & Se respetó la programación * & & & \\
\hline \multirow[t]{6}{*}{ instrucción } & Analfabeto & $-0,516-1,077$ & 0,05 & 0,002 \\
\hline & Primaria & $0,444-1,157$ & & \\
\hline & Secundaria & $0,956-1,350$ & & \\
\hline & Superior & $1,380-1,980$ & & \\
\hline & Atención rápida exámenes radiológicos * & & & \\
\hline & Analfabeto & $-0,948-0,718$ & 0,049 & 0,004 \\
\hline
\end{tabular}


Satisfacción del usuario externo de neurocirugía del Instituto Nacional de Ciencias Neurológicas, Lima - Perú, 2016

\begin{tabular}{|c|c|c|c|c|}
\hline Según & Variable predictiva & $\begin{array}{l}\text { Puntaje SERVQUAL } \\
\text { (IC del } 95 \% \text { ) }\end{array}$ & $\mathrm{Eta}^{2}$ & $\mathbf{p}$ \\
\hline & Primaria & $-0,477-0,269$ & & \\
\hline & Secundaria & $0,162-0,574$ & & \\
\hline & Superior & $0,392-1,020$ & & \\
\hline & Respeto a privacidad* & & & \\
\hline & Analfabeto & $-1,149-0,427$ & 0,063 & 0,000 \\
\hline & Primaria & $-0,600-0,105$ & & \\
\hline & Secundaria & $-0,164-0,226$ & & \\
\hline & Superior & $0,259-0,853$ & & \\
\hline & Comprendió la explicación del neurocirujano sobre su tratamiento* & & & \\
\hline & Analfabeto & $1,144-2,724$ & 0,059 & 0,001 \\
\hline & Primaria & $1,313-2,020$ & & \\
\hline & Secundaria & $0,994-1,384$ & & \\
\hline & Superior & $0,578-1,173$ & & \\
\hline & Los carteles o letreros son adecuados para orientar* & & & \\
\hline & Analfabeto & $-0,239-1,580$ & 0,060 & 0,001 \\
\hline & Primaria & $1,001-1,815$ & & \\
\hline & Secundaria & $0,407-0,857$ & & \\
\hline & Superior & $0,010-0,695$ & & \\
\hline \multirow[t]{16}{*}{ Procedencia } & Respeto a privacidad* & & & \\
\hline & Lima & $-0,517--0,167$ & 0,18 & 0,000 \\
\hline & Provincia & $0,501-0,912$ & & \\
\hline & Examen físico completo y minucioso* & & & \\
\hline & Lima & $-0,122-0,314$ & 0,06 & 0,000 \\
\hline & Provincia & $0,566-1,079$ & & \\
\hline & Comprendió la explicación del neurocirujano sobre su tratamiento* & & & \\
\hline & Lima & $1,414-1,774$ & 0,13 & 0,000 \\
\hline & Provincia & $0,499-0,923$ & & \\
\hline & Equipos disponibles y materiales para atención* & & & \\
\hline & Lima & $0,291-0,697$ & 0,058 & 0,000 \\
\hline & Provincia & $-0,336-0,141$ & & \\
\hline & Limpieza de sala de espera y comodidad* & & & \\
\hline & Lima & $1,131-1,515$ & 0,070 & 0,000 \\
\hline & Provincia & $0,428-0,879$ & & \\
\hline & ${ }^{*}$ Edad controlada o covariable & & & \\
\hline
\end{tabular}




\section{DISCUSIÓN}

La mayoría de los pacientes tuvieron cobertura del Sistema de Seguro Integral, incluso los que tuvieron intervenciones quirúrgicas. Esta es una variable que hay que tener en consideración al interpretar los resultados, pues aquellos que pagan por un servicio de salud podrían tener expectativas y percepciones diferentes en relación a los que tienen cobertura de un seguro público. Por lo tanto, la generalización de nuestros resultados a toda la población que se atiende en el Instituto Nacional de Ciencias Neurológicas debe ser considerada para una población con seguro distributivo.

La mayoría de pacientes incluidos en el presente estudio procedía de Lima. Ello es similar a la composición urbana del país, pero también muestra la dificultad de acceder a los servicios de salud de las zonas alejadas de la capital.

La investigación permitió determinar el grado de satisfacción de los usuarios externos de consulta de Neurocirugía del Instituto Nacional de Ciencias Neurológicas en el periodo de octubre- noviembre 2016 y enfatizar las percepciones de los mismos en cuanto a la calidad de atención recibida en dicha atención ${ }^{(18)}$.

Los usuarios externos mostraron un alto grado de insatisfacción debido a diferentes factores biológicos, ambientales, sociales, culturales, trato, tiempo de espera, demora en la atención, etc. Por lo tanto, es conveniente y oportuno identificar estos factores para elaborar acciones de mejora en la calidad de atención.

La medición del estudio se basó en el puntaje total del instrumento de la encuesta SERVQUAL, la cual presenta como valor final la diferencia entre las expectativas y las percepciones (menor de $0=$ Muy satisfecho, $0=$ Satisfecho, mayor que 0 y menor o igual a 2 = Insatisfacción leve y mayor que 2 = Insatisfacción moderada).

De nueve estudios previos, los de Fernández R. (2009) (19), Cuba M, Jurado G, Estrella E. (2011) (20), Shimabuku R, Huicho L, Fernández D, et al. (2010) (21) y la Oficina de Gestión de la Calidad del INCN 2012, 2013 y 2014, utilizaron, al igual que nuestra investigación , la encuesta SERVQUAL como instrumento de recolección de datos. Los estudios señalados se realizaron en Perú; en el caso de las investigaciones internacionales ${ }^{(22,23)}$ se trabajó con otros instrumentos como el SERVQHOS y el SEEUS.

En lo que concierne a las limitaciones del SERVQUAL como instrumento, podemos decir que, a pesar de su uso popular y aplicación generalizada, la encuesta ha sido objeto de una serie de críticas teóricas y operacionales. En el campo teórico, SERVQUAL (SQ) está basada en una inconformidad paradigmática porque establece la diferencia entre percepciones y expectativas, en lugar de un paradigma actitudinal. La encuesta SQ no mide características económicas ni condiciones psicológicas del paciente, se enfoca en el servicio ofertado y no en el resultado del servicio; en la atención y no en la resolución de la atención como resultado (tratado o curado). Respecto a su dimensionalidad, tiene 5 dimensiones que no necesariamente son universales y que pueden ser adaptadas o mejoradas de acuerdo al contexto. Desde el punto de vista operacional, la expectativa debe tomarse como polisémica, los consumidores tienen estándares en contraste a la evaluación de las expectativas, y SQ puede fallar en la medición de las expectativas. Respecto a los momentos de verdad (MV), la evaluación del SQ por los pacientes puede variar de MV a MV. Además, administrar dos instrumentos puede causar aburrimiento y confusión, lo que constituye una limitante que el investigador debe tratar de controlar para obtener buenos resultados y que no siempre es tomada en cuenta ${ }^{(24)}$.

En nuestro estudio encontramos que 170 usuarios, que representan el $54 \%$ del total, se encuentran insatisfechos con la atención recibida; y 144 usuarios, que corresponden al $46 \%$ de los encuestados, se encuentran satisfechos. Estos hallazgos son muy parecidos a estudios previos realizados en Perú (19-21,24).

De las dimensiones evaluadas en este estudio (fiabilidad, capacidad de respuesta, seguridad, empatía y aspectos tangibles), las que presentaron un alto grado de insatisfacción fueron empatía (64\%), fiabilidad (57 \%), y capacidad de respuesta (56\%); a diferencia de las otras dimensiones (seguridad y aspectos tangibles). Los resultados son muy similares a las investigaciones en otros hospitales de Perú (19-21,24).

Al considerar que la calidad de atención cobra cada vez mayor interés en la salud pública, y al reconocer que la atención adecuada satisface al paciente, se debe abordar, según el estudio, la dimensión más crítica, que es la empatía, lo que nos lleva a trabajar con el recurso humano en cuanto a sus habilidades blandas, para así poder mejorar este punto.

En el estudio realizado en la Unidad de Cuidados Intensivos del Instituto Nacional de Salud del Niño por Luján et al. en el año $2011^{(24)}$, en el que se utiliza también como herramienta a la encuesta SERVQUAL , se obtiene un 90,2\% de insatisfacción, que es mucho mayor al obtenido en nuestra investigación. En el referido estudio, la muestra fue de solo 105 familiares, mientras que en el nuestro es de 314 pacientes. A diferencia de nuestro trabajo, donde la insatisfacción fue $80,6 \%$; en este se describe que la dimensión con mayor insatisfacción, en adultos maduros 
fue la empatía, con $55,1 \%$.

También contamos con la investigación realizada por Acuña et al. ${ }^{25)}$, que evalúa el nivel de satisfacción del adulto mayor en la consulta externa del Hospital Edgardo Rebagliati Martins, y obtuvo un nivel de satisfacción del $65,6 \%$. Podemos objetivar que la satisfacción obtenida en este estudio es mayor al nuestro, pero hay diferencias respecto a los pacientes encuestados, como la edad y características distintas de los servicios, debido a que nuestro estudio se realizó en establecimientos del Ministerio de Salud (Minsa), y el estudio en comparación se ejecutó en EsSalud.

Como ya lo hemos dicho la primera causa de insatisfacción en esta investigación fue la dimensión empatía, que incluye aspectos como el trato amable, el interés que muestra el profesional de salud que atiende, y si el paciente llega a comprender la explicación brindada por el médico, tanto en su terapéutica como procedimientos a realizar.

La segunda dimensión con mayor porcentaje de insatisfacción es la fiabilidad. En este campo, el aspecto que provoca mayor desagrado es el tiempo de espera para ser atendido, hecho que se repite en diversos estudios y que nos debería hacer pensar sobre el mal sistema que se emplea para la atención de los pacientes. Se debe recalcar que el disgusto no es por el tiempo que transcurre entre la atención entre paciente y paciente, ya que para la mayoría de los usuarios es agradable que el médico se tome el tiempo necesario para escuchar y explicar cada detalle en la consulta, sino la demora en el inicio de la atención. Los médicos y demás trabajadores de salud deberían iniciar labores a las 8:00 a. m., sin embargo, a pesar que llegan a esa hora a sus establecimientos no empiezan con las consultas en el horario indicado.

Deberíamos tener en cuenta que elementos tan simples como la puntualidad y eficiencia de los trabajadores de caja, admisión y farmacia ayudarían a una mejor percepción de la atención de los establecimientos en estudio.

Otro estudio en el que también se utiliza a la encuesta SERVQUAL es el realizado en consultorios externos del hospital de la región Lambayeque en octubre del 2010 por Niño-Effio et al. (26). En este trabajo se encuentra un nivel de insatisfacción del 89,8\% valor mayor al nuestro $(80,6 \%)$, la dimensión con mayor insatisfacción fue la de seguridad con $93,5 \%$ y la de mayor satisfacción empatía con $55,1 \%$.

Como una limitación de análisis para la discusión, señalamos que no se encontraron artículos comparables y específicos acerca de la satisfacción de usuarios en consulta externa por neurocirugía en hospitales o institutos, ya sea en nuestro idioma o inglés, cuando realizamos la búsqueda con las palabras claves según los descriptores en ciencias de la salud (DeCS) "Satisfacción del Paciente", "Consultorios Médicos", "SERVQUAL", "Neurocirugía" , en las fuentes de información EBSCO, PROQUEST, ScienceDirect, JSTOR, PUBMED, LILACS, BIREME y WILEY; por lo que fue una restricción para profundizar y contrastar resultados según dimensiones global y específica del estudio.

En conclusión, la satisfacción global fue 19,4 \% (61) y la insatisfacción global fue en 80,6\% (253). En el 55,1\% (173) de adultos maduros hubo insatisfacción global. Respecto a la tangibilidad, el adulto maduro es el que presenta mayor insatisfacción con 36,6\% (115), del mismo modo para la fiabilidad con $13,3 \%$ (42), respuesta $46,8 \%$ (147), seguridad $14,9 \%$ (47), y empatía 55,1 \% (173). La media de las puntuaciones diferenciales entre expectativas y percepciones para todas las dimensiones fue 0,77 correspondiente a insatisfecho leve. Las medias de las puntuaciones diferenciales de las dimensiones específicas fueron las siguientes: fiabilidad 0,91 , capacidadvde respuesta 0,85 , seguridad 0,44 , empatía 1,03 , y aspectos tangibles 0,52 ; que son calificadas como un nivel insatisfecho leve.

\section{REFERENCIAS BIBLIOGRÁFICAS}

1. Munro-Faure L, Munro-Faure M. ¿Qué podemos aprender de los gurúes de la calidad? En: Munro-Faure L, Munro-Faure M. La calidad total en acción. Barcelona: Folio; 1994. p. 17-33.

2. Bayraktarogl G, Demirel BA. Testing the superiority and dimensionality of SERVQUAL vs. SERVPERF in higher education. Qual Manag J. 2010 Jan; 17(1): 47-59.

3. Cleary PD. Health care quality: Incorporating consumer perspectives. JAMA Netw Open. 1997 Nov; 278(19): 1608-12.

4. Cotlear D, Alvarado PB, Belsky L, Benavides L, Crouch L, Lavado PP, et al. ¿Cómo mejorar la educación, la salud y los programas antipobreza?. En: Cotlear D, editor. Un nuevo contrato social para el Perú: ¿cómo lograr un país más educado, saludable y solidario?. Lima: Banco Mundial; 2006. p. 86-7.

5. Evans L. Using SERVQUAL to Determine Veteran Healthcare Service Quality Profile with Recommendations. 2008; 1-114.

6. Parasuraman A, Berry LL, Zeithaml VA. Refinement and reassessment of the SERVQUAL scale. J Retailing. 1991; 67(4): 420-50.

7. Babakus E, Mangold WG. Adapting the SERVQUAL scale to hospital services: an empirical investigation. Health Serv Res. 1992 Feb; 26(6): 767-86.

8. Cabello E, Chirinos JL. Validación y aplicabilidad de encuestas SERVQUAL modificadas para medir la satisfacción de usuarios externos en servicios. Rev Med Hered. 2012 Abr-Jun; 23(2): 88-95.

9. Cabrera Moresco MA. Nivel de satisfacción del usuario externo atendido en el Tópico de Medicina del Servicio de Emergencia del Hospital Nacional Dos de Mayo, Lima, Noviembre 2015 [Tesis]. Lima: Universidad Ricardo Palma. Facultad de 
Medicina; 2016.

10. Ministerio de Salud. Dirección General de Salud de las Personas Lima - Perú. Encuesta de satisfacción de usuarios externos de servicios de salud. 2002: 1-21.

11. Instituto Nacional de Ciencias Neurológicas. Estadísticas 2015. Lima: Ministerio de Salud; 2015. Disponible en: http://www. incn.gob.pe/index.php/menu-estadi/294-estadistica-2015

12. Instituto Nacional de Ciencias Neurológicas. Boletín estadístico de actividades de salud. Lima: Ministerio de Salud; 2017. p. 1-85. Disponible en: http://www.incn.gob. pe/images/ESTADISTICAS/2017/BOLETIN\%20ESTADISTICO\%20 DE\%20ACTVIDADES\%20\%20DE\%20SALUD\%202017.pdf

13. Instituto Nacional de Ciencias Neurológicas. Informe de evaluación de la satisfacción de los usuarios externos de los servicios de consulta externa, emergencia y hospitalización del Instituto Nacional de Ciencias Neurológicas. Lima: Ministerio de Salud; 2015: 1-46.

14. Carvajal A, Centeno C, Watson R, Martínez M, Sanz Rubiales Á. ¿Cómo validar un instrumento de medida de la salud?. Anales Sist San Navarra. 2011 Ene-Abr; 34(1): 63-72.

15. Asubonteng P, McCleary KJ, Swan JE. SERVQUAL revisited: a critical review of service quality. J Serv Mark. 1996 Dec; 10(6): 62-81.

16. Kilbourne WE, Duffy JA, Duffy M, Giarchi G. The applicability of SERVQUAL in cross-national measurements of health-care quality. J Serv Mark. 2004 Dec; 18(7): 524-33.

17. Norma Técnica de Salud Categorías de establecimientos del Sector Salud. Resolución Ministerial $N^{\circ}$ 914-2010/MINSA. Ministerio de Salud; 2010. Disponible en: www.dgiem.gob. pe/wp-content/uploads/2013/01/pw48_rm546-2011-minsants021.pdf.

18. Instituto Nacional de Ciencias Neurológicas. Satisfacción del usuario externo. Lima: Ministerio de Salud; 2015. p. 1-46. Disponible en: http://www.incn.gob.pe/images/Calidad_ en_salud/Estudio_de_Satisfaccion_del_usuario_Externo INCN_2015.pdf

19. Fernández Coronado RO. Calidad de la atención y grado de satisfacción del paciente cardiaco transferido de provincia a la consulta externa de cardiología del INCOR 2009 [Tesis]. Lima: Universidad Nacional Mayor de San Marcos. Facultad de Medicina; 2014.

20. Cuba-Fuentes M, Jurado A, Estrella E. Evaluación del cumplimiento de los atributos de la Atención Primaria y grado de satisfacción de los usuarios de un establecimiento de primer nivel de atención. Rev Médica Hered. 2011; 22(1): 4-9.

21. Shimabuku R, Huicho L, Fernández D, Nakachi G, Maldonado $R$, Barrientos A. Niveles de insatisfacción del usuario externo en el Instituto Nacional de Salud del Niño de Lima, Perú. Rev Perú Med Exp Salud Pública. 2012 Oct-Dic; 29(4): 483-9.

22. De la Hoz Correa AP. Propuesta de aplicación de la escala SERVQUAL en el sector salud de Medellín. Revista CES Salud Pública. 2014; 5(2): 107-16.

23. Numpaque-Pacabaque A, Rocha-Buelvas A. Modelos SERVQUAL y SERVQHOS para la evaluación de calidad de los servicios de salud. Rev Fac Med. 2016; 64(4): 715-20.

24. Luján Donayre JH. Nivel de satisfacción según expectativas y percepciones de la calidad de servicio de salud en los usuarios externos de la unidad de cuidados intensivos del Instituto Nacional de Salud del Niño [Tesis]. Lima: Universidad Inca Garcilazo de la Vega. Facultad de Enfermería; 2011.

25. Acuña Zavala PD, Adrianzén Rodríguez SB, Almeyda Alvarado KA, Carhuancho Aguilar JR. Evaluación del nivel de satisfacción del adulto mayor entre 60 a 90 años en la consulta ambulatoria del Hospital Edgardo Rebagliati Martins. Rev Horiz Med. 2012; 12(3): $20-4$

26. Niño-Effio BS, Perales-Carrasco JCT, Chavesta-Manrique XP, Leguía-Cerna JA, Díaz-Velez C. Calidad de servicio en la consulta externa de un hospital de la región Lambayeque octubre 2010. Rev Cuerpo Méd HNAAA. 2012; 5(1): 5-9.

Fuentes de financiamiento:

Este artículo ha sido financiado por los autores.

Conflictos de interés:

Los autores declaran no tener ningún conflicto de interés.

Correspondencia:

Isabel Robles Hurtado

Dirección: Jr. Ancash 1271 - Barrios Altos. Lima, Perú.

Teléfono: (01) 4117700

Correo electrónico: isa_24021@hotmail.com

\section{Recibido: 10 de setiembre de 2018 . Evaluado: 19 de octubre de 2018. Aprobado: 17 de enero de 2019.}

( $)$ La revista. Publicado por Universidad de San Martín de Porres, Perú. (c) ${ }^{8}$ Licencia de Creative Commons Artículo en acceso abierto bajo términos de Licencia Creative Commons Atribución 4.0 Internacional. (http://creativecommons.org/licenses/by/4.0/)

\section{ORCID iDs}

Isabel Robles Hurtado

Ricardo Carreño Escobedo

Maritza Placencia Medina https://orcid.org/0000-0002-2779-8898 https://orcid.org/0000-0003-3707-2764 https://orcid.org/0000-0003-3624-3461 